\title{
Effects of Low Temperature Stress and Release on Photosynthetic Indexes of Greening Seedlings of Three Tree Species
}

\author{
Zhuomin Wang ${ }^{1, \text { a }}$, Jianxin $\mathrm{Xu}^{2, \mathrm{~b}}$, Li Xue ${ }^{3, \mathrm{c}_{*}}$, Tongtong Zhou ${ }^{4, \mathrm{~d}}$ \\ 1,3,4 College of Forestry, South China Agricultural University, Guangzhou, 510642, P. R. China \\ ${ }^{2}$ Shenzhen Tech and Ecology \& Environment CO., LTD, Shenzhen, Guangdong 518040, P. R. \\ China \\ a461584483@qq.com, b63930048@qq.com, cforxue@scau.edu.cn, d1152110287@qq.com \\ *Corresponding author: Li Xue (forxue@scau.edu.cn)
}

Keywords: greening seedling; low temperature stress and release; photosynthetic index; principal component analysis

Abstract. Photosynthetic characteristics of seedlings of Carallia brachiata, Ficus microcarpa and Phoebe zhennan were studied at $6^{\circ} \mathrm{C}$ low temperature stress using Artificial Climate Box. After continuous treatments of $0 \mathrm{~d}, 2 \mathrm{~d}, 4 \mathrm{~d}, 6 \mathrm{~d}$ and a recovery of $2 \mathrm{~d}$ in nature, the photosynthetic indexes of the seedlings were determined, and their cold-resistance ability was evaluated using principal component analysis. The results showed that with increasing low temperature stress time, the net photosynthetic rate, the stomatal conductance and the transpiration rate of the seedlings of three tree species decreased; the intercellular carbon dioxide concentration of $C$. brachiate increased followed by a decrease, whereas that of $F$. microcarpa and $P$. zhennan continuously increased; the stomatal limit of $C$. brachiata and P. zhennan continuously decreased, whereas $F$. microcarpa decreased followed by an increase. Two days after low-temperature release, the net photosynthetic rate, the transpiration rate and the stomatal limit of the seedlings of three tree species increased, whereas their intercellular carbon dioxide concentration decreased. The stomatal conductance of $C$. brachiata decreased, whereas that of $F$. microcarpa and $P$. zhennan increased. The comprehensive cold-resistance ability of photosynthetic indexes of the seedlings of three tree species was evaluated with principal component analysis, which decreased in the order of C. brachiata $>P$. zhennan $>F$. microcarpa.

\section{Introduction}

Low temperature is one of the significant factors that limit the geographic distribution of plants, it can cause plant photoinhibition [1], reduce the activity of antioxidase [2], destroy chloroplast structure [3], influence stomatal conductance [4] and reduce net photosynthetic rate [5].

Urban greening is one of the important strategies to protect natural environment, provide rest area and beautify the city, whereas low temperature is a vital limiting factor for the growth and geographical distribution of greening plants, therefore studying the cold-resistance of plants has an important significance. Previous studies have been focused on the effects of low temperature stress on cold resistance of vegetables and fruits [6], native species [7], grasses [8] and few tree species in Southern China [9]. Carallia brachiate, Ficus microcarpa and Phoebe zhennan are major greening and landscaping species of tropical and subtropical regions. However, the effect of low temperature stress on their photosynthetic physiology has remained unclear. Thus, the aim of this study was to examine low temperature effect on photosynthetic parameters of these seedlings.

\section{Materials and methods}

Study site. The study was conducted in the building of College of Forestry and Landscape Architecture, South Agricultural University, Guangzhou City. The study region has a subtropical monsoon climate with warm, rainy and adequate light. The annual average temperature, the average temperature of coldest month (January) and the hottest month (July) are $21.8^{\circ} \mathrm{C}, 13.3^{\circ} \mathrm{C}$ and $28.1^{\circ} \mathrm{C}$, 
respectively. The annual rainfall is $1714.4 \mathrm{~mm}$, mainly occurring from April to September, and the annual average relative humidity is $79 \%$.

Plant materials. One-year-old seedlings of C. brachiata, F. microcarpa and P. zhennanall were taken from Shenzhen Techand Ltd. The growth status of these seedlings was shown in Table 1.

Table 1 Growth status of three seedling types

\begin{tabular}{cccc}
\hline Tree species & Mean ground diameter $[\mathrm{cm}]$ & Mean Height $[\mathrm{cm}]$ & Crown width [cm] \\
Carallia brachiata & $0.48 \pm 0.02$ & $27.8 \pm 2.1$ & $22.1 \pm 0.8$ \\
Phoebe zhennan & $0.39 \pm 0.04$ & $20.2 \pm 1.2$ & $16.2 \pm 0.8$ \\
Ficus microcarpa & $0.79 \pm 0.6$ & $44.3 \pm 1.5$ & $20.7 \pm 0.9$ \\
\hline
\end{tabular}

Test method. In June 2014, the experimental seedlings with similar growth status were put into RXZ intelligent artificial climate chamber (Ningbo Jiangnan Instrument Factory). Lighting time was from 8:00 to $17: 00$ with a light intensity of $120 \mathrm{mmol}$ (photons) $\cdot \mathrm{m}^{-2} \cdot \mathrm{s}^{-1}$ and a relative humidity of $80 \% \sim 85 \%$. The seedlings were treated with $6 \pm 0.5^{\circ} \mathrm{C}$ low temperature (low temperature treatment 0 $\mathrm{d}$ as the control). Temperature of intelligent artificial climate box was reduced with $6^{\circ} \mathrm{C} \cdot \mathrm{h}^{-1}$. The temperature was maintained stability when it dropped to $6{ }^{\circ} \mathrm{C}$. Choosing five seedlings from each species at $0 \mathrm{~d}, 2 \mathrm{~d}, 4 \mathrm{~d}$ and $6 \mathrm{~d}$ after low temperature treatment, and six leaves from third to eighth functional leaves from the top of each seedling to be marked with label, the photosynthetic gas exchange parameters of these leaves were measured. Finally, the seedlings treated with low temperature of $6 \mathrm{~d}$ were moved out from the artificial climate chamber to restore growth for $2 \mathrm{~d}$ in natural environment, the photosynthesis parameters of the seedlings were measured.

Determination for index. Using Li-6400 portable photosynthesis system analyzer produced by LI-COR Company from US was used to determine net photosynthetic rate $\left(P_{n}\right)$, stomatal conductance $\left(G_{s}\right)$, transpiration rate $\left(T_{r}\right)$, intercellular $\mathrm{CO}_{2}$ concentration $\left(C_{i}\right)$ of the seedlings and their stomatal limitation value $\left(L_{s}\right)$ was calculated. Leaf chamber temperature was $20 \sim 25^{\circ} \mathrm{C}$, light intensity was $1000 \mu \mathrm{mol} \cdot \mathrm{m}^{-2} \cdot \mathrm{s}^{-1}, \mathrm{CO}_{2}$ concentration was $400 \mu \mathrm{mol} \cdot \mathrm{mol}^{-1}$ under the determined condition, and each leaf was measured three times. The formula of stomatal limitation $(L s)$ was as follows:

$L_{S}=(1-C i / C a)$.

where $\mathrm{Ca}$ was atmospheric $\mathrm{CO}_{2}$ concentration.

Data analysis. All statistical analyses were used Microsoft Excel and SAS8.1. After each index was standardized using range method, Principal component analysis was used for comprehensive evaluation. The standardized formula was as follow:

$$
X=1-\frac{x-x_{\min }}{x_{\max }-x_{\min }}
$$

where $X$ was the data after standardized, $x$ was the original data of eachn index, $x_{\min }$ was the minimum of original data, while $x_{\max }$ was the maximum of original data[10].

\section{The results and analysis.}

With increasing low temperature time, the net photosynthetic rate $\left(P_{n}\right)$ of the three species continuously decreased (Fig. 1). The Pn of the three species significantly increased $2 \mathrm{~d}$ after low-temperature release $(P<0.05)$. 

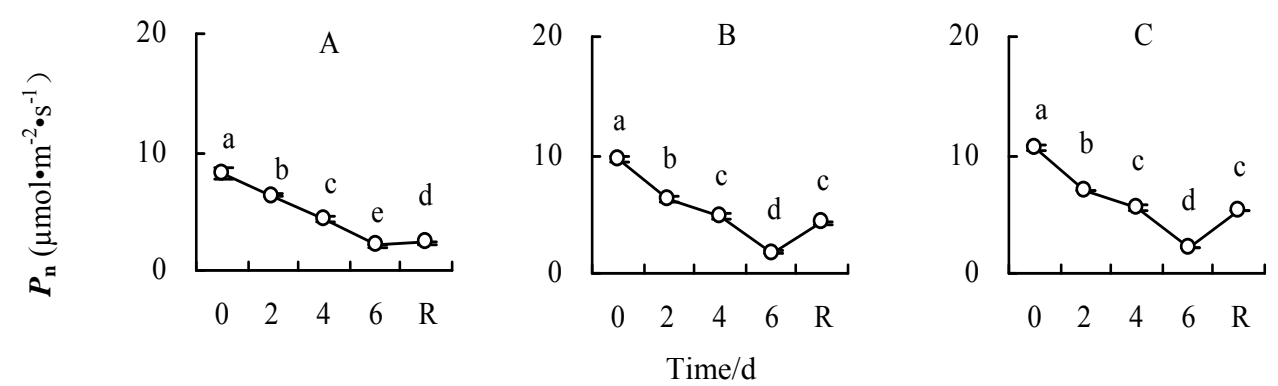

Fig. 1 Effect of low temperature stress on the net photosynthetic rate $\left(P_{\mathrm{n}}\right)$ in seedling leaves of the examined plants

A. Carallia brachiata; B.Ficus microcarpa; C. Phoebe zhennan

Significant differences between treatments were indicated by different letters above bars, $P<0.05$. The same below.

With increasing low temperature time, the stomatal conductance $\left(G_{s}\right)$ decreased sharply (Fig. 2). Two days after low-temperature release, the $G_{s}$ of C.brachiate decreased continuously, whereas F.microcarpa and P.zhennan significantly increased $(P<0.05)$.
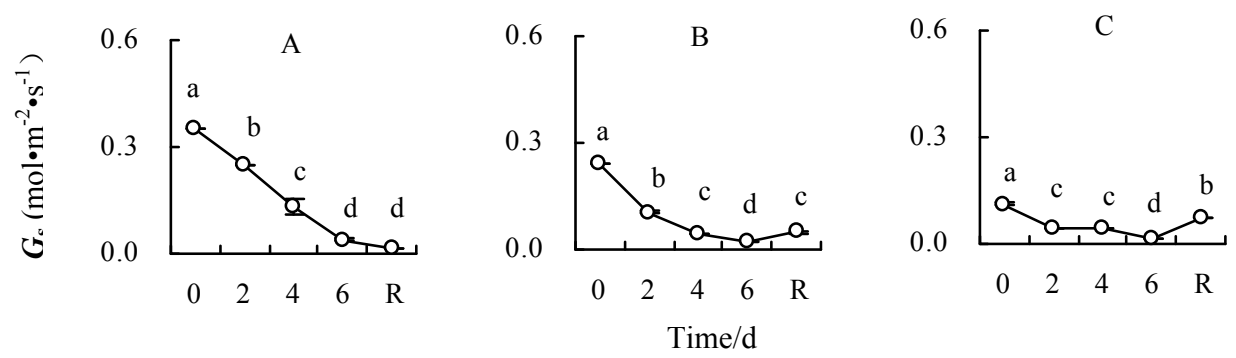

Fig. 2 Effect of low temperature stress on the stomatal conductance $\left(G_{\mathrm{s}}\right)$ in seedling leaves of the examined plants

A. Carallia brachiata; B. Ficus microcarpa; C. Phoebe zhennan

With increasing low temperature time, intercellular $\mathrm{CO}_{2}$ concentration $\left(C_{i}\right)$ of $C$. brachiate increased followed by a decrease, whereas F. microcarpa and $P$. zhennan continuously increased (Fig. $3)$. The $C_{i}$ of seedlings of the three species decreased $2 \mathrm{~d}$ after low-temperature release.
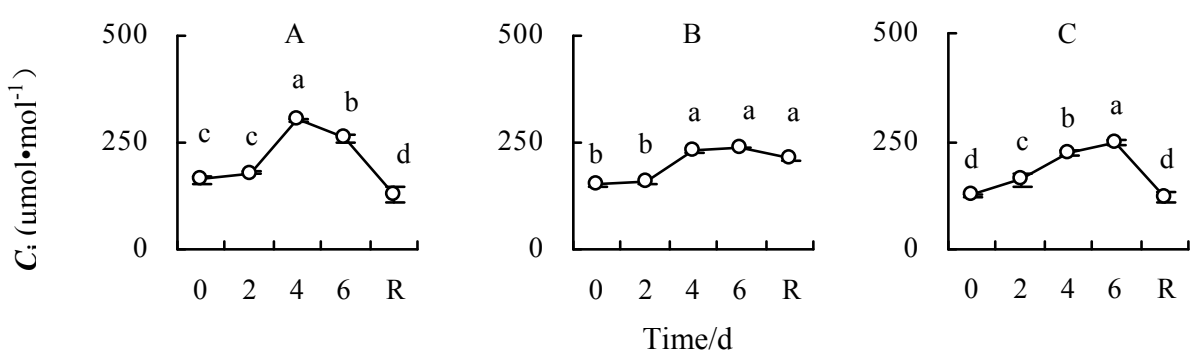

Fig. 3 Effect of low temperature stress on the Intercellular $\mathrm{CO}_{2}$ concentration $\left(C_{\mathrm{i}}\right)$ in seedling leaves of the examined plants

A. Carallia brachiata; B.Ficus microcarpa; C.Phoebe zhennan

With increasing low temperature time, the transpiration rate $\left(T_{r}\right)$ of seedlings of the three species continued to decline (Fig. 4). The Tr of seedlings of the three species increases significantly $2 \mathrm{~d}$ after low-temperature release $(P<0.05)$. 

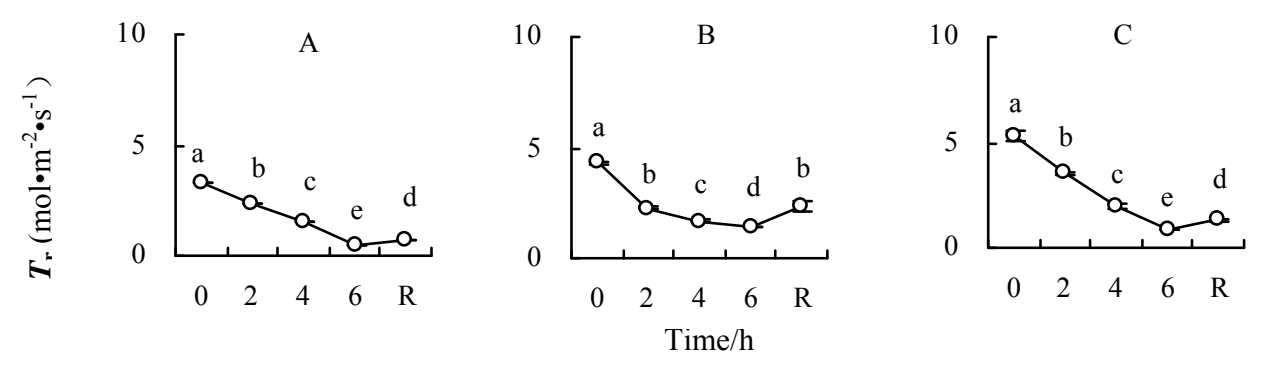

Fig. 4 Effect of low temperature stress on the transpiration rate $\left(T_{\mathrm{r}}\right)$ in seedling leaves of the examined plants

A. Carallia brachiata; B. Ficus microcarpa; C.Phoebe zhennan

During low temperature stress period, the stomatal limitation (Ls) of C. brachiate and P. Zhennan seedlings decreased continuously, whereas F. microcarpa decreased followed by an increase (Fig. 5). The Ls of seedlings of the three species increases significantly $2 \mathrm{~d}$ after low-temperature release $(P<0.05)$.
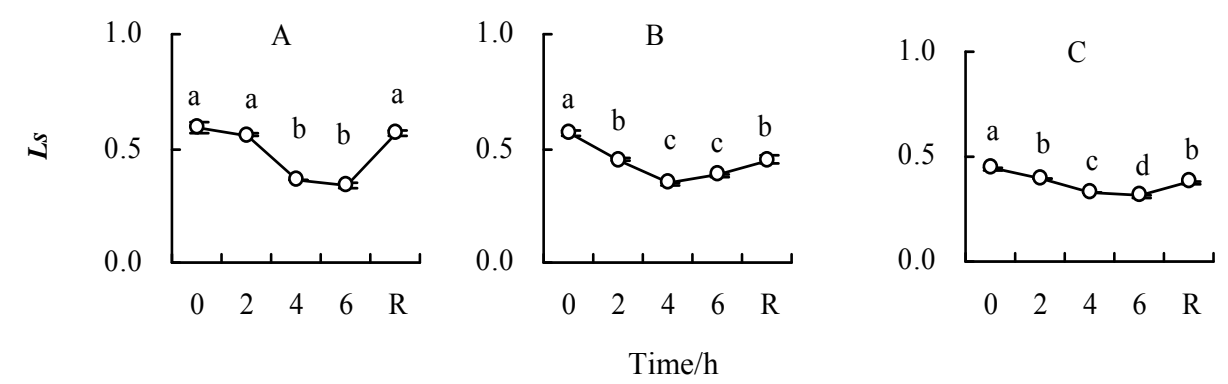

Fig. 5 Effect of low temperature stress on stomatal limit in seedling leaves of the examined plants A. Carallia brachiata; B.Ficus microcarpa; C.Phoebe zhennan.

Comprehensive evaluation of cold-resistance ability of the seedlings. The cold-resistance scores of the seedlings can be calculated by principal component analysis, and result indicated that the cold-resistance ability of the seedlings decreased in the order of C. brachiate $>P$. zhennan $>F$. microcarpa (Table 2).

Table 2 Comprehensive assessment values and orders of cold resistance of seedlings of three species

\begin{tabular}{ccc}
\hline \multicolumn{2}{c}{ Table 2 Comprehensive assessment values and orders of cold resistance of seedlings of three species } \\
\hline Testing species & Principal component scores & Score \\
\hline Carallia brachiata & 1.54 & 1 \\
Phoebe zhennan & 1.51 & 2 \\
Ficus microcarpa & 1.03 & 3 \\
\hline
\end{tabular}

\section{Conclusion.}

In this study, the net photosynthetic rate $\left(P_{n}\right)$, the stomatal conductance $\left(G_{s}\right)$ and the transpiration rate $\left(T_{r}\right)$ of the three species continued to decrease with increasing low temperature stress time. Because low temperature stress can lead to the imbalance of active oxygen metabolism of plants, destroy the structure of biofilm and chloroplast, affect intercellular $\mathrm{CO}_{2}$ concentration of leaves, thus it influences photosynthesis [5].

In general, the decrease of photosynthetic rate can be caused by stomatal limiting factor or non-stomatal limiting factor [11], which can be determined according to the change direction of $L_{\mathrm{s}}$ and $C_{i}$. If $C_{i}$ decrease and $L_{\mathrm{s}}$ increase, the reduction of $P_{n}$ is mainly resulted from stomatal limitation; on the contrary, that is mainly resulted from non-stomatal limitation [5]. After $4 \mathrm{~d}$ low temperature stress, the $C_{i}$ of $C$. brachiata and F. microcarpa had an increase, which indicated that the reduction of $P_{n}$ was mainly resulted from non-stomatal limitation. After $6 \mathrm{~d}$ low temperature stress, the $C_{i}$ of $C$. brachiate decreased, but its $L_{s}$ increased, illustrating that the decrease of $P_{n}$ was mainly resulted from 
stomatal limitation. Stomata closure inhibited the gas exchange and $\mathrm{CO}_{2}$ absorption of seedlings, which caused $P_{n}$ of C.brachiate to decrease. The $C_{i}$ of $P$. zhennan continued to reduce during the stress time, so decrease in $P_{n}$ was mainly resulted from non-stomatal limitation.

Two days after low-temperature release, the $P_{n}, G_{s}$ and $T_{r}$ of seedlings of the three species increased, whereas their $C_{i}$ decreased, suggesting that these seedlings have certain ability of cold resistance. The decrease of $C_{i}$ and the increase of $L_{s}$ of seedlings of the three species showed $P_{n}$ was mainly resulted from stomatal limitation just at the moment, and the decrease of $C_{i}$ resulted from partial closure of stomata.

According to the results of principal component analysis, the cold-resistance ability of seedlings of the three species decreased in the order of $C$. brachiata $>P$. zhennan $>F$. microcarpa, which means that $C$. brachiate can be given priority to choose, when experimental tree species were planted to cold regions.

\section{References}

[1] S. Takahashi and N. Murata: Trends in Plant Science Vol. 13 (2008), p. 178-182.

[2] R. Aroca, J.J. Irigoyen, A. Sanchez-Diaz: Plant Science Vol. 161 (2001), p. 719-726.

[3] H.A. Kratsch and R.R. Wise: Plant, Cell and Environment Vol. 23 (2000), p. 337-350.

[4] D. Eamus: Australian Journal of Plant Physiology Vol. 14 (1987), p. 331-339.

[5] S.H. Guo, L. Xue, R. Zhang and Z.Y. Yang: Journal of South China Agricultural University Vol. 33 (2012), p. 373-377. In Chinese.

[6] Z.Q. Yang, B. Zhang, J.B. Zhang, X.D. Peng, T.H. Zhang and J. Zhang: Journal of Natural Disasters Vol. 21 (2012), p. 168-174. In Chinese.

[7] Y.X. Yuan, Y. Wang, B.H. Pei and X.G. Jia: Acta Phytoecologiac Sinica 20(1996), p. 65-73. In Chinese.

[8] G.Z. Zhang and X.Y. Xiao: Bulletin of Botanical Research 17 (1997), p. 200-206. In Chinese.

[9] G.H. Liao: Eucalypt Science \& Technology 1 (1997), p. 5-10. In Chinese.

[10] G.C. Lu, J.X. Xu, L. Xue, R. Zhang, C.Q. Wu and Y.R. Shao: Journal of Central South University of Forestry \& Technology 34 (2014), p. 44-49. In Chinese.

[11]D.Q. Xu: Plant Physiology Communications 33 (1997), p. 241-244. In Chinese. 